\title{
Chemical complexity of mineral-fluid systems addressed by tools of statistical mechanics
}

\author{
I. KURGANSKAYA ${ }^{1 *}$ AND A. LUTTGE ${ }^{1}$
}

${ }^{1}$ MARUM \& FB5, Universität Bremen, 28359 Bremen,

Germany (*inna.kurganskaya@uni-bremen.de)

Mineral-fluid interactions in chemically heterogeneous environments constitute one of the major challenges in modern geochemistry. A large variety of possible chemical reactions and physical processes may occur at the mineralwater interface: dissolution, precipitation, crystal growth, ion and particle adsorption/desorption, as well as material transport. Correct physical descriptions of such systems at atomistic to nano scale can be addressed best by the tools of statistical mechanics. We use the Kinetic Monte Carlo (KMC) method to simulate complex reaction networks stochastically. Previously, we applied this method to study silicate [1,2] and carbonate mineral dissolution [3]. The influence of fluid

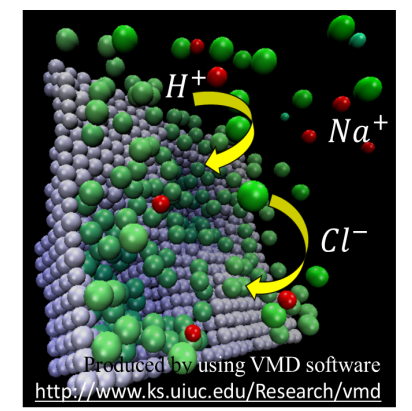
chemistry, e.g., $\mathrm{pH}$ and background electrolyte, onto surface site speciation is studied separately by Metropolis Monte Carlo in the Grand Canonical (GCMC) ensemble defined by chemical potentials of the ionic species. The coupled KMCGCMC model was sucessfully applied to address $\mathrm{pH}-$ dependence mechanisms for calcite dissolution [4]. We use this approach further to investigate mechanisms for saturation-state control of kinetics especially focusing on near-equilibrium processes. The problem is interesting and highly non-trivial according to the systematic studies of this dependence [5]. In this talk we deliver a summary of the results mentioned above and discuss our methodological concepts used to develop theory of crystal dissolution. A special attention is given to coupled dissolution/precipitation processes and their statistical characterization. We also relate this study to pore-scale mineral reaction kinetics where saturation-state control has critical importance.

[1] Kurganskaya \& Luttge (2013) J. Phys. Chem. C 117, 24894-24906. [2] Kurganskaya \& Luttge (2013) Geochim. Cosmochim. Acta 120, 545-560. [3] Kurganskaya \& Luttge (2016) J. Phys. Chem. 120 (12), 6482-6492. [4] Kurganskaya \& Churakov (2018) Phys. Chem. C 122, 29285-29297. [5] Luttge et al. (2019) Chem. Geol. 504, 216-235. 